\title{
Reference intervals for orotic acid in urine, plasma and dried blood spot using hydrophilic interaction liquid chromatography-tandem mass spectrometry ${ }^{\text {is }}$
}

\author{
Oceania D’Apolito a, ${ }^{\mathrm{a}}$, Daniela Garofalo ${ }^{\mathrm{a}, 1}$, Giancarlo la Marca ${ }^{\mathrm{b}, \mathrm{c}}$, Antonio Dello Russo ${ }^{\mathrm{d}}$, \\ Gaetano Corso ${ }^{\mathrm{a}, \mathrm{d}, *}$ \\ a Department of Biomedical Sciences, University of Foggia, Foggia, Italy \\ ${ }^{\mathrm{b}}$ Department of Pharmacology, University of Florence, Italy \\ ' Mass Spectrometry Lab, Meyer Children's Hospital, Florence, Italy \\ d Department of Biochemistry and Medical Biotechnologies, University Federico II of Naples, Naples, Italy
}

\section{A R T I C L E I N F O}

\section{Article history:}

Received 19 July 2011

Accepted 27 September 2011

Available online $\mathrm{xxx}$

\section{Keywords:}

Reference intervals

Orotic acid

Dried blood spot

Tandem mass spectrometry

\begin{abstract}
A B S T R A C T
Orotic acid $(\mathrm{OA})$, a marker of hereditary orotic aciduria, is usually used for the differential diagnosis of some hyperammonemic inherited defects of urea cycle and of basic amino acid transporters. This study was aimed to establish age related reference intervals of $\mathrm{OA}$ in urine, and for the first time in plasma, and dried blood spot (DBS) from 229 apparently healthy subjects aged from three days to 40 years. The quantification of OA was performed by a previously implemented method, using a stable isotope dilution with 1,3-[ $\left.{ }^{15} \mathrm{~N}_{2}\right]$-orotic acid and hydrophilic interaction liquid chromatography-tandem mass spectrometry (HILIC-MS/MS). The method has proved to be sensitive and accurate for a quantitative analysis of OA also in DBS and plasma. According to previous studies, urinary OA levels $(\mathrm{mmol} / \mathrm{mol}$ of creatinine) decrease significantly with age. The upper limits (as 99th \%ile) were of 3.44 and 1.30 in groups aged from three days to 1 year (group 1 ) and from 1 year to 12 years (group 2), respectively; in teenagers (from 13 to 19 years; group 3 ) and adults (from 20 to 40 years; group 4 ) urinary levels became more stable and the upper limits were of 0.64 and 1.21 , respectively. Furthermore, OA levels in DBS $(\mu M)$ also resulted significantly higher in subjects of group 1 (upper limit of 0.89 ) than in subjects of groups 2,3 and 4 (upper limits of $0.24,0.21$, and 0.29 , respectively). OA levels in plasma $(\mu \mathrm{M})$ were significantly lower in subjects of group 3 (upper limit of 0.30 ) than in subjects of groups 1,2 , and 4 (upper limits of 0.59 , 0.48 , and 0.77 , respectively). This method was also employed for OA quantification in plasma and DBS of 17 newborns affected by urea cycle defects, resulting sensitive and specific enough to screen these disorders.
\end{abstract}

(C) 2011 Elsevier B.V. All rights reserved.

\section{Introduction}

Orotic acid (OA; 1,2,3,6-tetrahydro-2,6-dioxo-4pyrimidinecarboxylic acid; uracil-6-carboxylic acid) is a metabolic marker of the hereditary orotic aciduria, a pyrimidine synthesis defect caused by uridine-5'-monophosphate (UMP) synthase deficiency [1] (OMIM 258900 and 258920), but the most valuable use of OA determination is for the differential diagnosis of ornithine transcarbamoylase (OTC, OMIM 311250) deficiency, the most common form of a urea cycle defect. Increased excretion of urinary

\footnotetext{
This paper is part of the special issue "LC-MS/MS in Clinical Chemistry", Michael Vogeser and Christoph Seger (Guest Editors).

* Corresponding author at: Dipartimento di Scienze Biomediche, Facoltà di Medicina e Chirurgia - Università di Foggia, Viale L. Pinto 1, 71122 Foggia, Italy. Tel.: +39 0881 588055; fax: +390881588037.

E-mail address: g.corso@unifg.it (G. Corso).

1 These authors contributed equally to this work.
}

OA is also observed in the other inborn errors of the urea cycle, as argininosuccinate synthetase deficiency (ASS, citrullinemia type I, OMIM 215700), argininosuccinate lyase deficiency (ASL, argininosuccinic aciduria, OMIM 207900), and arginase deficiency (ARG, argininemia, OMIM 207800). OA in urine also increases in defects of genes encoding some cationic amino acid transporters such as the mitochondrial ornithine transporter 1 deficiency (ORNT 1; HHH syndrome, OMIM 238970), and the lysine transporter $\mathrm{y}^{+}$LAT1 (SLC7A7; LPI, lysinuric protein intolerance, OMIM 222700), which impair the intestinal absorption and renal re-absorption of basic amino acids, respectively. In these conditions the reduced blood levels of ornithine and/or of arginine slow down the rate of urea cycle [2,3]. OA determination is helpful for the differential diagnosis of hyperammonemia disorders which cannot be readily diagnosed by amino acid chromatography, thus reducing the need for enzyme determination in tissue biopsies.

OA excretion increases also as a result of drugs, such as allopurinol and 6-azauridine, that appears to inhibit the distal part of pyrimidine synthesis pathway. Once OA reaches the blood, it is 
efficiently cleared by the kidney; there is evidence for secretion by the tubules, as well as loss by filtration [4]. OA may even be successfully analyzed in pieces of urine-impregnated filter paper $[5,6]$ and mailed to a central laboratory; today, a variety of analytical techniques provide satisfactory results [2]. Analysis of urinary OA provides an integrated picture of OA production over time, rather than the snapshot that is provided by analysis of a single plasma sample [7].

Although the $\mathrm{OA}$ in urine represents worldwide the reference test for differential diagnosis and for the monitoring of disorders above mentioned, patients affected by urea cycle defects also show elevated OA levels in plasma or blood $[3,4,8]$, and only few affected patients with these disorders have been carefully investigated analyzing OA in blood or plasma. Increased concentration of OA in plasma and blood has been found in ASL [4], and OTC defects [9].

We recently implemented a fast and sensitive hydrophilic interaction LC (HILIC)-MS/MS method for the quantitative determination of $\mathrm{OA}$ in urine, plasma, and dried blood spot (DBS) requiring only minimal sample preparation and providing a short run time. Using this method, OA levels in plasma and DBS from a patient affected by citrullinemia were at least fifty times higher than normal controls [8]. These rare data make it difficult to define safety limits of OA in blood and its usefulness as a biomarker of disease; moreover, reference intervals in blood of healthy humans are completely lacking.

Today, tandem MS represents a powerful tool for quantitative analysis of metabolites in biological fluids, and, in particular, the metabolic analysis in DBS by MS/MS represents the reference method for the expanded newborn screening of inborn errors of metabolism; therefore, the analysis of OA in DBS might represent a useful test for the diagnosis of classical orotic acidurias, or as a second tier test to confirm urea cycle defects.

The purpose of this study was to determine reference intervals for OA concentrations in urine, plasma, and DBS from apparently healthy children and adults, divided into four groups aged from three days to 40 years, and to assess the effect of age. In addition, we report the results of OA in DBS and plasma from some patients affected by different inborn errors of urea cycle.

\section{Materials and methods}

\subsection{Chemicals}

The analytical solvents of HPLC grade ACN, and ammonium acetate were obtained from J.T. Baker (Deventer, The Netherlands). OA standard was purchased from Sigma-Aldrich (Steinheim, Germany), and [1,3- $\left.{ }^{15} \mathrm{~N}_{2}\right]-\mathrm{OA}$, used as internal standard (IS), was purchased from Cambridge Isotope Laboratories (Andover, MA, USA). Filter paper for DBS preparation was of grade 903 and was purchased from Whatman GmbH (Dassel, Germany).

\subsection{Samples collection and population study}

Urine, plasma, and whole blood samples were referred to our laboratory (Clinical Biochemistry Laboratory of University Hospital of Foggia, Italy) for general laboratory tests. The collection of samples, for the establishment of reference intervals, was made between January 2010 and March 2011. Disease controls were selected from those of the newborn screening center at A. Meyer Children's Hospital, Florence (Italy). The samples were divided according to patient age into four groups: group 1 comprised 51 babies (20 females and 31 males; age, from three days to 1 year); urines were collected from all, and blood for plasma and DBS preparation were collected from 24 (9 females and 15 males). Furthermore, other 20 DBS samples were selected from unaffected patients ( 12 females and 8 males; age, 3-16 days) who had already been analyzed for metabolic diseases. Group 2 comprised 40 children ( 17 females and 23 males; age, $1-12$ years); group 3 comprised 40 teenagers ( 18 females and 22 males; age, 13-19 years); group 4 comprised 78 adults ( 55 females and 23 males; age, 20-40 years). In addition, another group ( $\mathrm{n}^{\circ} 5$ ) comprised 17 newborns ( 7 females and 10 males, from which 11 plasma and 6 DBS were obtained) affected by ASS deficiency ( $n=11)$, OTC deficiency $(n=2)$, ASL deficiency $(n=2)$, and CPS deficiency $(n=2)$, diagnosed on clinical, biochemical, and/or genetic grounds. All the samples analyzed in this study were reserve materials that were not needed for further diagnostic investigations, thus avoid taking extra materials or more volume of sample from patients.

The selection of normal samples was based on the normality of the following laboratory markers: glucose, urea, creatinine, uric acid, aspartate transaminase, alanine transaminase, gammaglutamyl-transferase, and hemoglobin. Aliquots of urine, plasma, and whole blood samples from apparently healthy individuals, not affected by urea-cycle disorders, were used to assess reference intervals of $\mathrm{OA}$.

\subsection{Specimen preparation and analysis}

\subsubsection{Creatinine measurement}

Urinary creatinine concentration was determined by a commercial kit, based on the Folin's method with the Jaffé reaction [10], on the automated instrument AU2700 (Beckman Coulter, Inc., Brea, CA). The concentration of $\mathrm{OA}$ is expressed as a ratio with urinary creatinine concentration ( $\mathrm{mmol} / \mathrm{mol}$ of creatinine) to take into account the variations of urinary volume among subjects, this procedure is commonly used in clinical biochemistry.

\subsubsection{DBS, plasma, and urine preparation}

DBS samples were prepared from EDTA blood spotting at least $30 \mu \mathrm{L}$ on filter paper and stored in desiccant sealed plastic bags at $-20^{\circ} \mathrm{C}$ until analysis. Plasma, obtained from EDTA blood, and urine samples were stored at $-20^{\circ} \mathrm{C}$ until analysis. A disc of $6 \mathrm{~mm}$ was punched from DBS and the OA was extracted by adding $10 \mu \mathrm{L}$ of an aqueous solution of IS $(5 \mu \mathrm{M}), 20 \mu \mathrm{L}$ of water, and $120 \mu \mathrm{L}$ of ACN with ammonium acetate $(5 \mathrm{mM})$. After mixing, the solution was incubated for $20 \mathrm{~min}$ at $30^{\circ} \mathrm{C}$, then $2 \mu \mathrm{L}$ of supernatant were analyzed. Twenty microliters of plasma sample were mixed with $20 \mu \mathrm{L}$ of IS solution, and the solution was diluted with $260 \mu \mathrm{L}$ of ACN with ammonium acetate $(4.6 \mathrm{mM})$. The mixture was vortexed, and allowed to stand for $5 \mathrm{~min}$ at room temperature and, after centrifugation at $3000 \times g$ for $5 \mathrm{~min}, 2 \mu \mathrm{L}$ of supernatant were analyzed. The urine samples were filtered to remove bacteria and any particulate material (membrane filter of $0.22 \mu \mathrm{m}$; Millex-GV, Millipore, France) and then diluted 1:10 with water before the analysis. Twenty microliters of diluted urine were mixed with $20 \mu \mathrm{L}$ of IS solution and diluted with $260 \mu \mathrm{L}$ of ACN with ammonium acetate $(4.6 \mathrm{mM})$, mixed and allowed to stand for $5 \mathrm{~min}$ at room temperature; then $2 \mu \mathrm{L}$ of the extract were injected.

\subsection{3. $L C-M S / M S$ analysis}

Urine, plasma, and DBS samples were analyzed by HILIC-MS/MS performed according to the method previously described [8]. Briefly, chromatography was performed by a column packed with sub-2 $\mu \mathrm{m}$ particles (Zorbax RX-SIL, $1.8 \mu \mathrm{m}, 50 \mathrm{~mm} \times 2.1 \mathrm{~mm}$ id, Agilent Technologies, USA), and OA was eluted by an isocratic mobile phase constituted of ACN/water (90/10) containing $4 \mathrm{mM}$ of ammonium acetate. The MS detection was performed by electrospray ionization and compounds were monitored using a multiple reaction monitoring (MRM) in negative mode of the following transitions: $m / z 155>111$ for $\mathrm{OA}$, and $m / z 157>113$ for $\left[1,3-{ }^{15} \mathrm{~N}_{2}\right]-\mathrm{OA}$ 
Table 1

Mean values, within-day, and between-day precision (CV\%) and accuracy (\%) obtained measuring OA in plasma, dried blood spot, and urine quality controls.

\begin{tabular}{|c|c|c|c|c|c|c|c|}
\hline \multirow{2}{*}{$\begin{array}{l}\text { Concentration of } \\
\text { added } \mathrm{OA}(\mu \mathrm{M})\end{array}$} & \multirow[t]{2}{*}{ Expected value } & \multicolumn{3}{|l|}{ Within-day $(n=6)$} & \multicolumn{3}{|c|}{ Between-day $(n=12)$} \\
\hline & & $\begin{array}{l}\text { Measured mean } \\
\text { values }\end{array}$ & Precision (CV\%) & Accuracy (\%) & $\begin{array}{l}\text { Measured mean } \\
\text { values }\end{array}$ & Precision (CV\%) & Accuracy (\%) \\
\hline \multicolumn{8}{|l|}{ Plasma } \\
\hline 0 & $\mathrm{~N} / \mathrm{A}$ & 0.25 & 13.2 & $\mathrm{~N} / \mathrm{A}$ & 0.27 & 18.5 & $\mathrm{~N} / \mathrm{A}$ \\
\hline 2.5 & 2.8 & 3.06 & 3.2 & 9.5 & 3.22 & 8.0 & 15.0 \\
\hline 15 & 15.2 & 14.7 & 3.8 & -3.4 & 14.6 & 8.2 & -4.1 \\
\hline \multicolumn{8}{|l|}{ DBS } \\
\hline 0 & $\mathrm{~N} / \mathrm{A}$ & 0.12 & 9.6 & $\mathrm{~N} / \mathrm{A}$ & 0.12 & 14.1 & $\mathrm{~N} / \mathrm{A}$ \\
\hline 2.5 & 2.6 & 2.55 & 2.8 & -2.8 & 2.69 & 13.6 & 3.6 \\
\hline 15 & 15.1 & 13.4 & 0.8 & -11.1 & 14.0 & 17.3 & -7.4 \\
\hline \multicolumn{8}{|l|}{ Urine $^{\mathrm{a}}$} \\
\hline 0 & $\mathrm{~N} / \mathrm{A}$ & 6.1 & 2.2 & $\mathrm{~N} / \mathrm{A}$ & 6.4 & 8.8 & $\mathrm{~N} / \mathrm{A}$ \\
\hline 5 & 11 & 12.3 & 1.9 & 11.6 & 12.8 & 5.8 & 16.5 \\
\hline 25 & 30.8 & 33.7 & 2.7 & 9.5 & 35.0 & 9.7 & 13.6 \\
\hline 150 & 154.3 & 149.1 & 1.9 & -3.4 & 166.3 & 7.2 & 7.8 \\
\hline
\end{tabular}

N/A: not applicable.

a Urine samples were diluted 1:10 before analysis.

internal standard. Both OA and its IS eluted at $2.00 \mathrm{~min}$, and the total run time was of $4 \mathrm{~min}$.

Plasma and DBS concentrations were calculated on calibration curves prepared using enriched plasma and blood in a concentration range from 0.1 to $25 \mu \mathrm{M}$. Blood calibration points were spotted on filter paper and analyzed. The urinary concentrations were calculated using a calibration curve prepared with aqueous standard solutions in a concentration range from 0.78 to $25 \mu \mathrm{M}$. The linearity of calibration curves was estimated by evaluation of coefficient of correlation $(r)$ that ranged among analytical batches from 0.997 to 0.999 for water, from 0.994 to 0.997 for plasma, and from 0.993 to 0.998 for DBS.

Quality control (QC) was carried out to evaluate within-day and between-day precision and accuracy. In each analytical batch QC specimens were analyzed together with real samples. An aliquot of blank pool of blood, plasma, and urine was used as low QC level only for precision evaluation, two aliquots were spiked with OA standard to obtain QCs at concentration of 2.5 and $15 \mu \mathrm{M}$ for plasma and blood, while for urine three aliquots of the normal pool were spiked with OA to obtain QCs at concentration of 5, 25, and $150 \mu \mathrm{M}$. The QC aliquots were stored as the clinical samples until use.

\subsection{Statistical analysis}

The acquired data were processed using the QuanLynx software (v. MassLynx 4.0, Waters) to calculate calibration curves, QCs, and sample concentrations. Statistical parameters were obtained by Microsoft Excel software (v. 11.0, Microsoft) and/or with KaleidaGraph software (version 4.1.1). ANOVA analysis was performed using the Bonferroni post-test correction. The data of each group were tested for distribution and, if a Gaussian distribution was obtained, the mean value was used to set a limit at the mean $\pm 2 \mathrm{SD}$; the values outside the limit were considered to be outliers and rejected. When the data sets showed non-Gaussian distributions, a robust statistical method was used for the detection of outliers. This robust statistical technique uses distribution-free inter-quartile range (IQR) to set a Tukey fence $[11,12]$. The data were sorted into ascending sequence to obtain the lower and upper quartile point, $Q 1$ and $Q 3$, respectively. The difference between $Q 3$ and $Q 1(Q 3-Q 1)$ is named IQR. The Tukey fence is the range from $Q 1-1.5 *$ IQR to $Q 3+1.5^{*} \mathrm{IQR}$. The values outside the Tukey fence were considered to be outliers and removed. After the elimination of outliers, the data sets were re-tested for distribution, and if a Gaussian distribution was obtained, the mean value was used to establish a limit at the mean \pm 2 SD. Instead, if a non Gaussian distribution was obtained the data sets were described using median, range, and percentiles. The values outside the limit of $1 \mathrm{st}$ \%ile and of 99th \%ile were considered to be outliers and rejected.

\section{Results}

\subsection{Quality control results}

QC samples were analyzed during the assays of patient samples. Within-day precision (CV\%) and accuracy (\%) were calculated by analyzing each level of QC samples 6 times in the same analytical run. The expected value was calculated adding OA value in blank sample to the added amount of OA standard, corrected by sample volumes used to prepare the mixture (Table 1 ). The OA precision (CV\%) on three QC levels ranged from 3.2 to 13.2 for plasma, from 0.8 to 9.6 for DBS, and from 1.9 to 2.7 for urine, while the accuracy (\%) ranged from -3.4 to 9.5 for plasma, from -11.1 to -2.8 for DBS, and from -3.4 to 11.6 for urine. The between-day precision and accuracy were calculated by analyzing each level of QC samples once a day for 12 working days. The precision (CV\%) was estimated from 8.0 to 18.5 for plasma, from 13.6 to 17.3 for DBS, and from 5.8 to 9.7 for urine. The accuracy (\%) ranged from -4.1 to 15.0 for plasma, from -7.4 to 3.6 for DBS, and from 7.8 to 16.5 for urine (Table 1).

\subsection{Population study results}

The descriptive statistic of OA in plasma, DBS and urine of reference population is reported in Table 2. Urinary OA raw data of each group showed a non-Gaussian distribution; therefore, all data were elaborated by the robust statistic method to identify and to reject the outliers above the high Tukey fence. The values excluded were $2 / 51$ (3.9\%) from group 1, 3/40 (7.5\%) from group 2, 5/40 (12.5\%) from group 3, and 3/78 (3.8\%) from group 4. After the elimination of outliers, data sets were re-tested for distribution and a Gaussian distribution was obtained for groups 1,2 , and 3; instead, the group 4 still showed a non Gaussian distribution. However, parametric and non-parametric results in each group are very close; instead, comparing urinary excretion of OA among the groups, it was evident that the levels in group 1 were at least two times higher than in groups 2, 3, and 4 . The analysis of variance among groups showed significant differences between group 1 vs 2 , group 1 vs 3 , and group 1 vs 4 $(p<0.0001)$. We also correlated the age of patients and the urinary 
Table 2

Descriptive statistic of age-related orotic acid concentrations in plasma, dried blood spot, and urine from unaffected subjects.

\begin{tabular}{|c|c|c|c|c|}
\hline & \multicolumn{4}{|c|}{ Population study (age intervals, years) } \\
\hline & Group $1(<1)$ & Group $2(1-12)$ & Group 3 (13-19) & Group $4(20-40)$ \\
\hline \multicolumn{5}{|l|}{ Plasma $(\mu \mathrm{M})$} \\
\hline$(n)$ & $(23)$ & $(40)$ & (39) & $(73)$ \\
\hline Mean (SD) & $0.30(0.12)$ & $0.30(0.09)$ & $0.15(0.07)^{\mathrm{a}}$ & $0.28(0.16)$ \\
\hline Mean $\pm 2 S D$ & $0.06-0.54$ & $0.12-0.48$ & $0.01-0.29$ & $0.0-0.60$ \\
\hline Median & 0.27 & 0.27 & 0.13 & 0.22 \\
\hline Min-max & $0.12-0.60$ & $0.15-0.48$ & $0.03-0.31$ & $0.06-0.82$ \\
\hline 1st-99th\%ile & $0.13-0.59$ & $0.16-0.48$ & $0.04-0.30$ & $0.09-0.77$ \\
\hline \multicolumn{5}{|l|}{ DBS $(\mu \mathrm{M})$} \\
\hline$(n)$ & $(43)$ & $(40)$ & (39) & $(77)$ \\
\hline Mean (SD) & $0.38(0.24)^{b}$ & $0.12(0.06)$ & $0.11(0.04)$ & $0.14(0.05)$ \\
\hline Mean $\pm 2 S D$ & $0.0-0.86$ & $0.0-0.24$ & $0.03-0.19$ & $0.04-0.24$ \\
\hline Median & 0.35 & 0.10 & 0.10 & 0.13 \\
\hline Min-max & $0.07-0.91$ & $0.04-0.25$ & $0.04-0.22$ & $0.05-0.31$ \\
\hline 1st-99th\%ile & $0.07-0.89$ & $0.04-0.24$ & $0.04-0.21$ & $0.07-0.29$ \\
\hline \multicolumn{5}{|c|}{ Urine ( $\mathrm{mmol} / \mathrm{mol}$ of creatinine) } \\
\hline$(n)$ & $(49)$ & $(37)$ & $(35)$ & $(75)$ \\
\hline Mean (SD) & $1.65(0.80)^{\mathrm{c}}$ & $0.63(0.26)$ & $0.39(0.12)$ & $0.50(0.25)$ \\
\hline Mean $\pm 2 S D$ & $0.05-3.25$ & $0.11-1.15$ & $0.15-0.63$ & $0.0-1.00$ \\
\hline Median & 1.49 & 0.60 & 0.39 & 0.45 \\
\hline Min-max & $0.40-3.47$ & $0.22-1.39$ & $0.17-0.65$ & $0.01-1.27$ \\
\hline 1st 99th\%ile & $0.49-3.44$ & $0.23-1.30$ & $0.18-0.64$ & $0.13-1.21$ \\
\hline
\end{tabular}

ANOVA with Bonferroni's post test.

a $p<0.0001$ group 3 vs group 1 , group 2 , and group 4 .

b $p<0.0001$ group 1 vs group 2, group 3 , and group 4 .

c $p<0.0001$ group 1 vs group 2, group 3 , and group 4 .

OA excretion and, as reported in Fig. 1, there is a significant negative polynomial correlation $(p<0.0001)$.

Plasma OA raw data showed a non-Gaussian distribution for group 1,3 , and 4 ; therefore, after the rejection of outliers above the high Tukey fence, the values excluded were $1 / 24$ (4.2\%) from group 1, none from group 2, 1/40 (2.5\%) from group 3, and 5/78 (6.4\%) from group 4 . After the elimination of outliers, data sets were re-tested for Gaussian distribution, the group 4 still showed a non Gaussian distribution. Also for plasma, the parametric and nonparametric results are very close in each group (Table 2 ). The group 3 showed OA plasma levels lower than group 1, group 2, and group 4. Fig. 2 reports the dot-plot of plasma OA levels in all groups, and

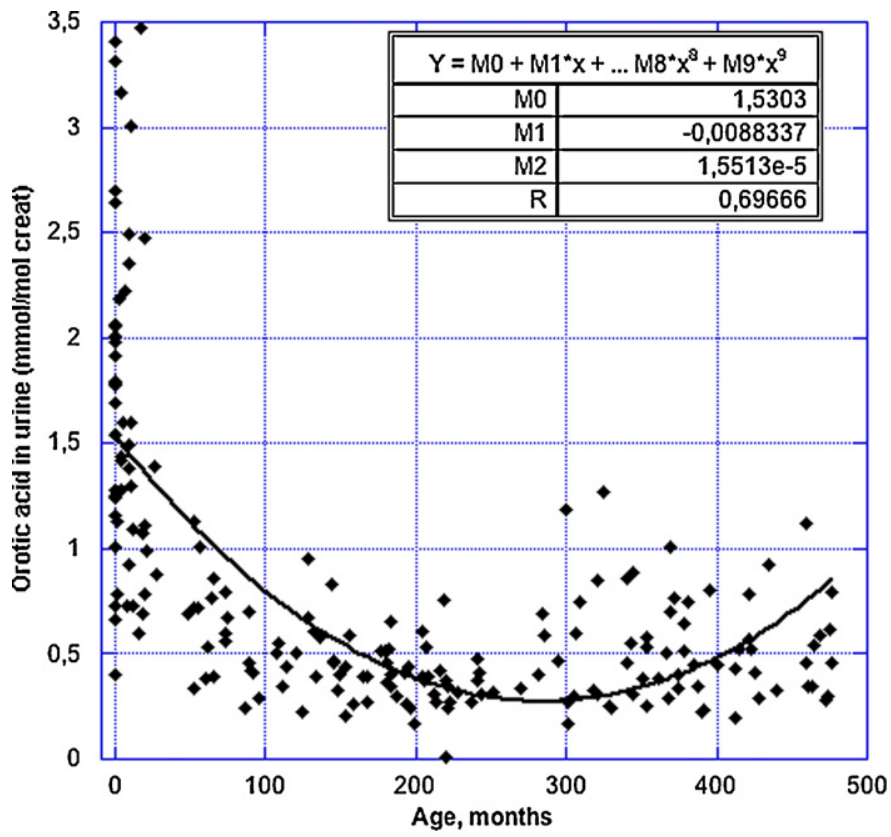

Fig. 1. Scatter-plot of reference urinary orotic acid values in groups 1-4 vs age (months). the analysis of variance among groups showed significant differences between group 3 vs group 1, group 3 vs 2, and group 3 vs 4 $(p<0.0001)$.

OA raw data in DBS showed a non-Gaussian distribution for group 1, 3, and 4; therefore, after the rejection of outliers above the high Tukey fence, the values excluded were $1 / 44$ (2.3\%) from group 1 , none from group $2,1 / 40(2.5 \%)$ from group 3 , and $1 / 78$ (1.3\%) from group 4 . After the elimination of outliers, data sets were re-tested for Gaussian distribution, which still was abnormal for group 4. Also for DBS, the parametric and non-parametric results are very close in each group (Table 2 ). Group 1 showed OA DBS levels higher than groups $2-4$. Fig. 3 reports the dot-plot of OA in DBS of all groups, and the analysis of variance among groups showed

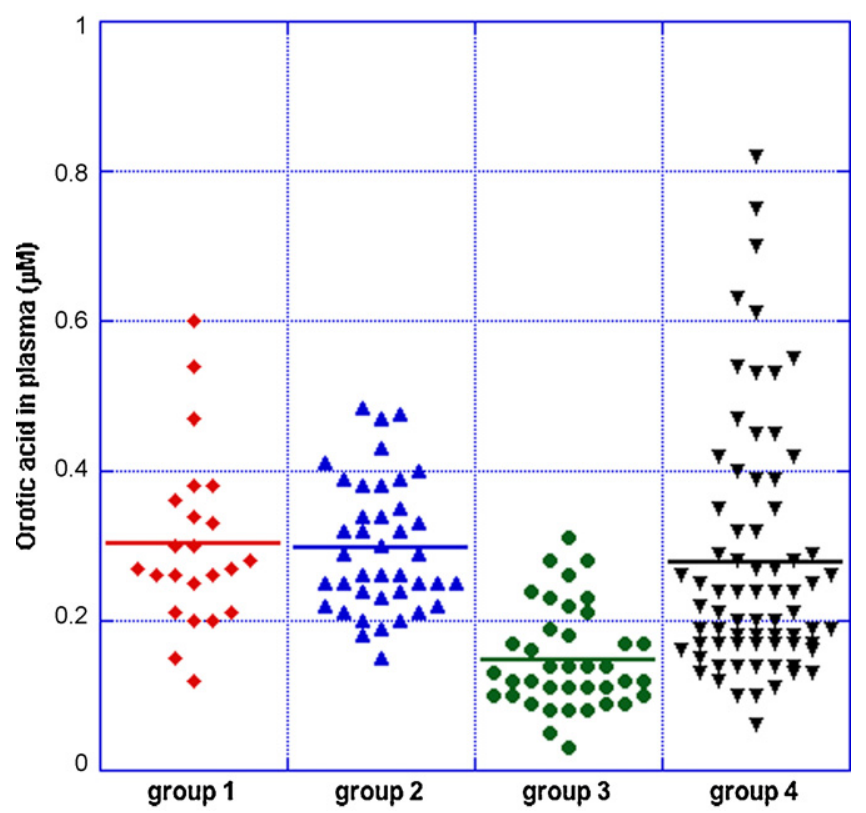

Fig. 2. Dot-plot of plasmatic orotic acid values in all reference groups. 


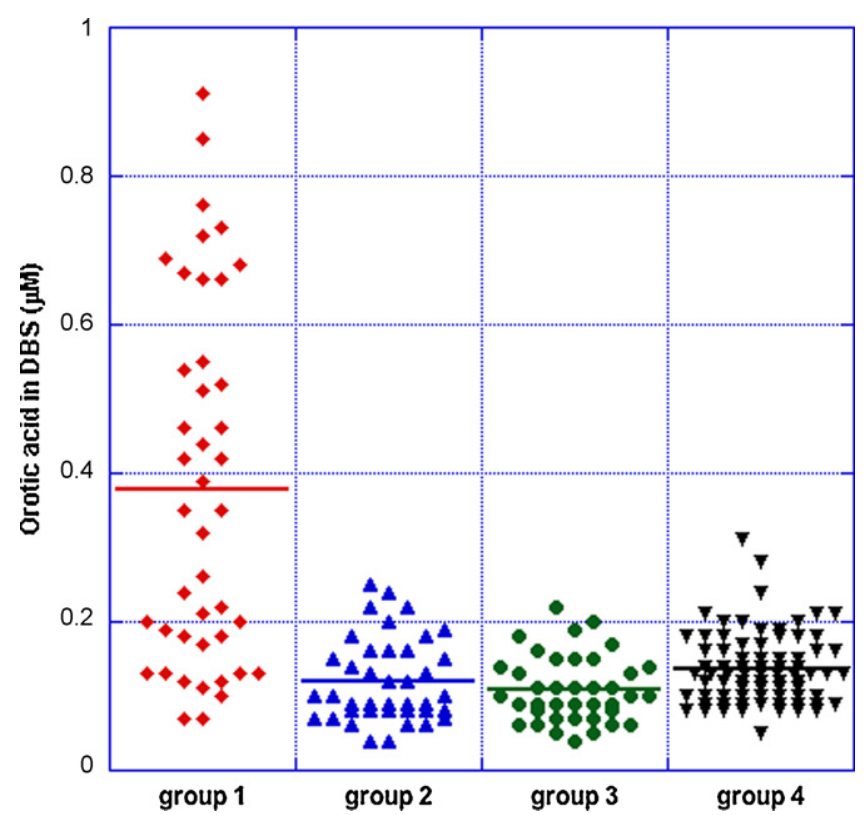

Fig. 3. Dot-plot of DBS orotic acid values in all reference groups.

significant differences between group 1 vs group 2, group 1 vs 3 , and group 1 vs 4 ( $p<0.0001)$.

Results from affected patients (group 5) are reported in Table 3. OA levels $(\mu \mathrm{M})$ ranged from 0.12 to 117 in plasma and from 12.8 to 46.6 in DBS of patients with ASS deficiency, from 4.8 to 86.3 in DBS of patients with OTC deficiency, and from 0.02 to 0.03 in plasma of patients with ASL deficiency during therapeutic treatment. Two patients with CPS deficiency showed OA levels of 0.28 in plasma and of 0.35 in DBS. In addition, the urinary excretion of OA ( $\mathrm{mmol} / \mathrm{mol}$ of creatinine) measured at moment of diagnosis in ASS patients \# 1 , and \# 2 was of 1.34 , and 1.63 , respectively.

\section{Discussion}

The main purpose of this study was to establish age related reference intervals of OA in DBS, plasma, and urine in unaffected

Table 3

Orotic acid levels in plasma and DBS samples of affected patients.

\begin{tabular}{|c|c|c|c|c|}
\hline \multirow{2}{*}{$\begin{array}{l}\text { Patient } \\
\text { samples }\end{array}$} & \multirow{2}{*}{$\begin{array}{l}\text { Age } \\
\text { (days) }\end{array}$} & \multirow[t]{2}{*}{ Defects } & \multicolumn{2}{|c|}{ Orotic acid $(\mu \mathrm{M})$} \\
\hline & & & Plasma & DBS \\
\hline $\mathrm{mf} \# 1$ & 4 & ASS-I & 0.17 & \\
\hline $\mathrm{mf} \# 2$ & 4 & ASS-I & 0.12 & \\
\hline $\mathrm{mf} \# 3$ & 3 & CPS & & 0.35 \\
\hline $\mathrm{mf} \# 5$ & 7 & CPS & 0.28 & \\
\hline $\mathrm{mf} \# 6$ & 4 & ASS-I & & 46.5 \\
\hline $\mathrm{mf} \# 7$ & 4 & ASS-I & 62.0 & \\
\hline $\mathrm{mf} \# 10$ & 4 & ASS-I & 6.1 & \\
\hline $\mathrm{mf} \# 11$ & 4 & ASL $^{*}$ & 0.03 & \\
\hline $\mathrm{mf} \# 12$ & 3 & OTC & & 86.3 \\
\hline $\mathrm{mf} \# 13$ & 4 & $\mathrm{ASL}^{\mathrm{a}}$ & 0.02 & \\
\hline $\mathrm{mf} \# 14$ & 3 & OTC & & 4.83 \\
\hline $\mathrm{mf} \# 15$ & 4 & ASS-I & & 46.6 \\
\hline $\mathrm{mf} \# 16$ & 4 & ASS-I & & 12.8 \\
\hline $\mathrm{mf} \# 17$ & 4 & ASS-I & 15.0 & \\
\hline $\mathrm{mf} \# 18$ & 4 & ASS-I & 1.0 & \\
\hline $\mathrm{mf} \# 19$ & 4 & ASS-I & 60.1 & \\
\hline fg \# 39 & 5 & ASS-I & 116.8 & \\
\hline Reference & Group 1 & & $0.13-0.59$ & $0.07-0.89$ \\
\hline intervals & $<1$ year & & & \\
\hline
\end{tabular}

a Both patients were under therapeutic treatment. children, teenagers, and adults. Both parametric and nonparametric results have been reported. The urinary values for unaffected patients suggested a significant but continuous negative polynomial correlation of OA concentrations ( $\mathrm{mmol} / \mathrm{mol}$ of creatinine) with age in children from three days up to adults (Fig. 1). In agreement with previous reports this trend is likely due to the increase of creatinine excretion with age [5,13-16]. We defined the limits of OA reference intervals between the 1 st \%ile and 99th \%ile, although in clinical practice the 99th \%ile limit is mostly of interest. Urinary OA limits in unaffected children between three days of birth and 1 year (group 1) would be from 0.49 to $3.44 \mathrm{mmol} / \mathrm{mol}$ of creatinine, and in group 2, between 1 year and 12 years, the limits would be from 0.23 to $1.30 \mathrm{mmol} / \mathrm{mol}$ of creatinine. In unaffected teenagers, between 13 and 19 years (group 3), the limits would be from 0.18 to $0.64 \mathrm{mmol} / \mathrm{mol}$ of creatinine, and in adults, between 20 and 40 years (group 4 ), would be from 0.13 to $1.21 \mathrm{mmol} / \mathrm{mol}$ of creatinine. The levels of urinary $\mathrm{OA}$ in group 1 are significantly higher than group 2, 3, and 4 . The age period from birth to 12 months (group 1) is more critical for the detection of many inborn pathologies. Instead, the variations of OA levels among groups 2, 3, and 4 appear relatively small and not statistically significant.

The 1 st and 99th \%ile of OA in plasma of unaffected children of group 1 would be from 0.13 to $0.59 \mu \mathrm{M}$, and in the group 2 would be from 0.16 to $0.48 \mu \mathrm{M}$. In unaffected teenagers, the limits would be from 0.04 to $0.30 \mu \mathrm{M}$, and in adults, from 0.09 to $0.77 \mu \mathrm{M}$. The levels of plasma OA in group 3 are significantly lower than other groups $(1,2$, and 4$)$, and the variations of OA levels among groups 1,2 , and 4 were not statistically significant.

The 1 st and 99th \%ile of OA in DBS of unaffected children of group 1 would be from 0.07 to $0.89 \mu \mathrm{M}$, and in the group 2 would be from 0.04 to $0.24 \mu \mathrm{M}$. Limits of group 3 would be from 0.04 to $0.21 \mu \mathrm{M}$, and in adults from 0.07 to $0.29 \mu \mathrm{M}$. The levels of plasma OA in group 1 are significantly higher than other groups (2-4). Instead, the variations of OA levels among groups 2,3, and 4 appear very small and not statistically significant. DBS and normalized urinary OA levels of group 1 both show significantly higher OA levels than other groups.

Assessment of urinary $\mathrm{OA}$ is facilitated by its remarkable stability. Normal human urine contains trace amounts of OA [17] and, as previously reported by us [8] and confirmed in this work, the compound is quantifiable at sub-micromolar level also in plasma and blood of unaffected patients.

The early colorimetric methods for OA determination in urine were simple and rapid, yet non-selective, which may give rise to false-positive results. OA reference intervals obtained by colorimetric methods $[18,19]$ are wider than those obtained by our method, and also by gas chromatography-mass spectrometry (GC-MS) methods $[5,16,20]$, and by ion-exchange chromatography methods with UV detection [13,14]. Unfortunately, each of these last two methods have difficulties and limitations, are time consuming and not adaptable as screening methods.

To date, since Ito et al. [21] described a LC-ESI-MS/MS method for a large number of urinary metabolites, including OA, others have proposed a more specific, high-throughput, sensitive stable-isotope-dilution LC-ESI-MS/MS method for the determination of urinary $\mathrm{OA}$ and intermediate metabolites in urine specimens $[6,15,22]$. Even though reference intervals of urinary OA obtained by LC-ESI-MS/MS are comparable to those reported in this study by HILIC-ESI-MS/MS, our reference limits are narrower and well overlapped with those obtained by stable isotope GC-MS methods $[5,16]$. In the method reported here, the OA from DBS, plasma, and urine was eluted from column pumping an isocratic mixture of solvents, which improves the reproducibility of retention time and the sensitivity; moreover, no interferences were observed during an analytical batch of 100 samples.

For the first time we report the reference intervals in plasma and DBS from apparently healthy subjects aged from three days to 40 
years, demonstrating that this method allows a quantitative analysis of $\mathrm{OA}$ also in these biological fluids. In addition, this method has been employed to analyze samples from patients affected by four different defects of urea cycle. Levels of OA in plasma and DBS from patients affected by ASS $(n=9 / 11)$ and OTC $(n=2 / 2)$ deficiencies were significantly over the 99th \%ile of our reference intervals; to note that only two patients affected by ASS deficiency (plasma samples \# 1 and \# 2) showed normal values, an expected result because at moment of sample collection they also showed normal excretion of orotic acid in urine (1.34, and $1.63 \mathrm{mmol} / \mathrm{mol}$ of creatinine, respectively). Whereas, two patients affected by CPS, a defect without orotic aciduria, and two patients affected by ASL under therapeutic treatment showed normal and low OA levels, respectively. Therefore, these findings suggest that determination of $\mathrm{OA}$ in plasma and DBS could be used as an additional test to screen several metabolic diseases, even though further evaluation should be performed on a higher number of positive samples.

\section{Conclusions}

Although the frequency of disorders presenting orotic aciduria is low, a fast and accurate analysis of OA using a small and easily transportable sample, such as DBS, should be useful and clinically relevant, in particular for newborn screening.

\section{Acknowledgements}

We are grateful to colleagues Maria Assunta Schiavone and Clelia Scarano (Clinical Biochemistry Laboratory, UniversityHospital of Foggia, Italy), and Monica Gelzo (University Federico II of Naples) for their valuable collaboration. The authors also thank the University of Foggia for funding (Premialità PRIN 2008).

\section{References}

[1] L.H. Smith, M. Sullivan, C.M. Huguley, J. Clin. Invest. 40 (1961) 656.

[2] C. Salerno, C. Crifò, J. Chromatogr. B 781 (2002) 57.

[3] M.E. Brosnan, J.T. Brosnan, J. Nutr. 137 (2007) 1656S

[4] J.O. Sass, D. Skladal, Pediatr. Nephrol. 13 (1999) 912.

[5] M.T. McCann, M.M. Thompson, I.C. Gueron, M. Tuchman, Clin. Chem. 41 (1995) 739.

[6] A.B.P. van Kuilenburg, H. van Lenthe, M. Loffler, A.H. van Gennip, Clin. Chem. 50 (2004) 2117.

[7] J. Rajantie, Pediatr. Res. 15 (1981) 115.

[8] O. D’Apolito, D. Garofalo, G. Paglia, A. Zuppaldi, G. Corso, J. Sep. Sci. 33 (2010) 966.

[9] H.R. Yoon, Arch. Pharm. Res. 30 (2007) 387.

[10] O. Folin, J. Biol. Chem. 17 (1914) 475.

[11] J.W. Tukey, Exploratory Data Analysis, Addison-Wesley, Reading, MA, 1977, p. 506.

[12] P.S. Horn, L. Feng, Y. Li, A.J. Pesce, Clin. Chem. 47 (2001) 2137.

[13] C. Bachaman, J.P. Colombo, J. Clin. Chem. Clin. Biochem. 18 (1980) 293.

[14] M. Asai, S. Sumi, K. Kidouchi, H. Imaeda, H. Togari, Y. Wada, Pediatr. Int. 42 (2000) 499.

[15] M.S. Rashed, M. Jacob, M. Al-Amoudi, Z. Rahbeeni, M.A.D. Al-Sayed, L. AlAhaidib, et al., Clin. Chem. 49 (2003) 499.

[16] J. Chen, M.J. Bennett, Methods Mol. Biol. 603 (2010) 445.

[17] M. Lotz, H.J. Fallon, L.H. Smith, Nature 197 (1963) 194.

[18] M.L. Harris, V.G. Oberhoizer, Chin. Chem. 26 (1980) 473.

[19] D. El Khiari, N. Tebib, N. Kaabachi, M. Feki, Y. Ben Hamouda, A. Mebazaa, Arch. Pediatr. 5 (1998) 15.

[20] M. Rimoldi, P. Bergomi, A. Romeo, S. Diflinato, J. Inherit. Metab. Dis. 17 (1994) 243.

[21] T. Ito, A.B.P. van Kuilenburg, A.H. Bootsma, A.J. Haasnoot, A. van Cruchten, Y. Wada, et al., Clin. Chem. 46 (2000) 445.

[22] S. Hartmann, J.G. Okun, C. Schmidt, C.D. Langhans, S.F. Garbade, P. Burgard, et al., Clin. Chem. 52 (2006) 1127. 